\title{
EXOTIC SOLUTIONS TO THE SOLAR NEUTRINO PROBLEM AND SOME IMPLICATIONS FOR LOW ENERGY SOLAR NEUTRINO EXPERIMENTS ${ }^{a}$
}

\author{
HIROSHI NUNOKAWA \\ Instituto de Física Gleb Wataghin, Universidade Estadual de Campinas, UNICAMP \\ 13083-970 Campinas SP, Brazil \\ E-mail: nunokawa@ifi.unicamp.br
}

\begin{abstract}
In this talk, I review, from the phenomenological point of view, solutions to the solar neutrino problem, which are not provided by the conventional neutrino oscillation induced by mass and flavor mixing, and show that they can provide a good fit to the observed data. I also consider some simple implications for low energy solar neutrino experiments.
\end{abstract}

\section{Introduction}

It is considered that observed data coming from atmospheric neutrino experiments 1 are compelling evidence of putrino oscillation indicating the presence of neutrino mass and flavor mixing el $^{3}$. This is now being confirmed by the ongoing K2K experiment 4 . Results of the solar neutrino experiments 5 are also supporting such mass induced neutringoscillations hypothesis, either through the matter enhanced MSW mechanism 6 or through the vacuum oscillation 3 . I will call these explanations "standard solutions" to the solar neutrino problem (SNP) as they are based only on neutrino mass and flavor mixing, the most natural extension of the standard model. Althogh not yet confirmed by other experiment, the LSND data are also indicating neutrino mass and mixing.

On the other hand, several alternative scenarios, which can explain these observatipn-withqut invoking neutrino mass and/or flavor mixing, have been proposed $8,9,16,11,12,13,14$ and some of them are not yet excluded. I will call them "non-standard" or "exotic" solutions, as they are theoretically less motivated compared to the standard solutions. In this talk, from the phenomenological point of view, I will review such non-standard solutions to the SNP which are providedby the neutrino conversion induced by resonantsnin-flavor precession $(\mathrm{RSFP})$ 8, non-standard neutrino interactions (NSNI) 6, 20.12 .12 , and violation of the equivalence principle (VEP) 13 and show that they can provide a good fit to the solar neutrino data. In the end, I will try to consider some possible implications for low energy solar neutrino experiments. For non-standard explanations of the atmospheric neutrino observations, see Ref. 44 for a review.

${ }^{a}$ Talk presented at International Workshop on Low Energy Solar Neutrinos (LowNu2), 4-5, December, 2000, Tokyo, Japan 


\section{Resonant Spin-Flavor Precession}

Let me start with the solution to the SNP induced by neutrino magnetic moment, as I think it less exotic in the sense that this solution does require neutrino masses in contrast to the other two solutions I will discuss in the next sections. If neutrinos have transition magnetic moment among different flavors, they can undergo spin-flavor precession (SFP) 15 in the presence of magnetic field. Moreover, in matter, such SFP can be resonantly enhanced in the same fashion as in the case of the MSW effect 6 . Such resonant conversion or RSFP can occur between $\nu_{e L}\left(\equiv \nu_{e}\right)$ and $\nu_{\mu, \tau R}^{c}\left(\equiv \bar{\nu}_{\mu, \tau}\right)$ for Majorana neutrinos and $\nu_{e L}$ and $\nu_{\mu, \tau R}$ for Dirac neutrinos $\left(\nu_{\mu, \tau R}\right.$ is a electroweak singlet neutrino). In order to have RSFP conversion, neutrinos must have different masses as in the case of the MSW effect, to satisfy the resonance condition $\mathrm{B}$.

A nice feature of this mechanism is, as stressed in Ref.16, that it can give the appropriate energy dependent suppressions of neutrino fluxes needed to account well for the solar neutrino data, i.e., it can provide strong suppression for ${ }^{7}$ Be neutrinos, weak suppression for $p p$ neutrinos and moderate suppression for ${ }^{8} \mathrm{~B}$ neutrinos. See Ref.17 for recent analyses on this mechanism

Here I present some updated analysis of our previous work 18 , for the case of Majorana neutrinos for $\nu_{e} \leftrightarrow \bar{\nu}_{\mu}$ (or $\bar{\nu}_{\tau}$ ) channel, taking one particular magnetic field profile used in our previous work 18 , which has the triangle shape concentrated in the solar convective zone (profile 3 in Ref. 18 ), as it can provide a very good fit to the solar neutrino data. Fixing the shape of the magnetic field profile, a fit to the solar neutrino data目 is performed by varying $\Delta m^{2}$ and the overall normalization of the magnetic field, or the average value, $\langle B\rangle$.
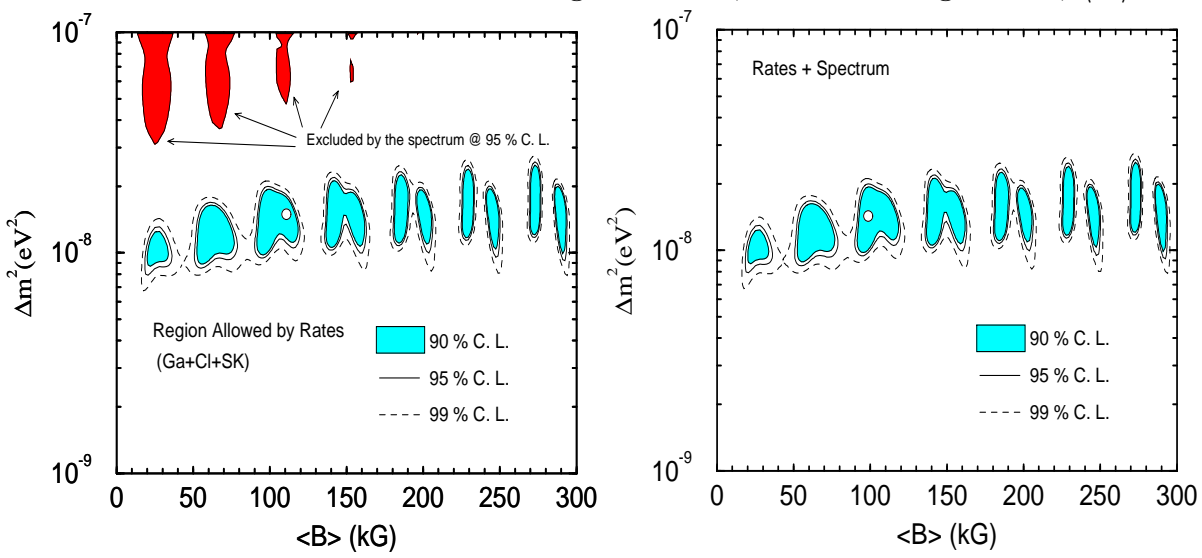

Fig. 1: In the left panel the parameter region allowed by the rates as well as the excluded parameter region by the SK spectrum are presented and in the right panel the combined allowed region is shown. 
I present in Fig. 1 the region allowed by total rates only, the SuperKamiokande (SK) spectrum only and the combined case. For definiteness, I fix the magnitude of neutrino magnetic moment $\mu_{\nu}$ to $10^{-11} \mu_{B}$, where $\mu_{B}$ is the Bohr magneton, which is still allowed by laboratory experiments 19 . Note, however, that the same results are obtained for different values of $\mu_{\nu}$ as long as the product $\mu_{\nu}\langle B\rangle$ is kept to be the same value. It is found that within the allowed region from the total rates, no strong distortion of the spectrum is expected, which is in good agreement with the current experimental situation. I show in Fig. 2 the expected SK recoil electron spectrum using the best fitted

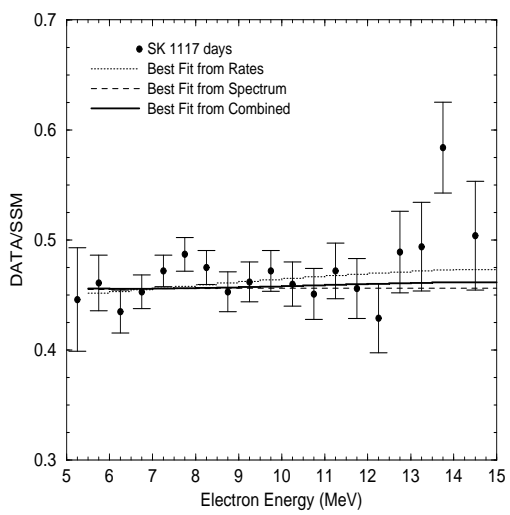

Fig. 2: Expected SK recoil electron spectrum using the best fitted parameters from the total rates, spectrum only and combined analysis. parameters. We see that no strong distortion is expected. It is found that for the total rates, $\chi_{m i n}^{2}=0.17$ for 2 degrees of freedom (DOF) and for the spectrum, $\chi_{\min }^{2}=13.6$ for $15 \mathrm{DOF}$, and for the combined $\chi_{\text {min }}^{2}=18.3$ for $24 \mathrm{DOF}$, where the contribution from SK zenith angle dependence (6 bins) was included though the RSFP mechanism does not induce any significant distortion of the zenith angle dependence. I conclude that RSFP mechanism can explain well the solar neutrino data provided that the product of neutrino magnetic moment and the strength of the solar convective zone magnetic field satisfies $\mu_{\nu} \cdot\langle B\rangle \gtrsim 10^{-11} \mu_{B} \cdot O(10) \mathrm{kG}$.

\section{Non-Standard Neutrino Interactions}

Next let me consider the solution based on resonantsoniersion induced by non-standard neutrino interactions (NSNI) in matter $9.10,11,122,20,21$. Here I will consider the phenomenological approach by simply assuming the existence of a tree-level process $\nu_{\alpha}+f \rightarrow \nu_{\beta}+f$ with an amplitude $\epsilon_{\alpha \beta} \sqrt{2} G_{F}$, where $\alpha$ and $\beta$ are flavor indices, $f$ stands for the interacting elementary fermion $(d, u$ quark or electron) and $\epsilon_{\alpha \beta}$ is considered to be free parameter, which characterize the strength of NSNI. Here I consider such NSNI induced only by $d$ or $u$-quark since if they are induced only by electrons, no resonant conversion can occur and the fit to the total rates is not so good 22 .

In the presence of such NSNI neutrino evolution equation in matter for the system of two massless neutrinos, $\nu_{e}-\nu_{x}(x=\mu, \tau)$, is given as 10 :

$$
i \frac{d}{d r}\left[\begin{array}{l}
\nu_{e} \\
\nu_{x}
\end{array}\right]=\sqrt{2} G_{F}\left[\begin{array}{cc}
n_{e}(r) & \epsilon n_{f}(r) \\
\epsilon n_{f}(r) & \epsilon^{\prime} n_{f}(r)
\end{array}\right]\left[\begin{array}{l}
\nu_{e} \\
\nu_{x}
\end{array}\right],
$$


where, $\epsilon \equiv \epsilon_{e x}$ and $\epsilon^{\prime} \equiv \epsilon_{x x}-\epsilon_{e e}$. Due to the presence of $\epsilon^{\prime}$ term, a MSW-like resonant conversiop can occur (when $n_{e}(r)=\epsilon^{\prime} n_{f}(r)$ is satisfied) even if neutrinos are massless 20 The crucial point which makes this mechanism a viable solution to the SNP23, despite the fact that the conversion probability itself is completely energy independent (see Eq. (11)), is that different production distributions of neutrinos can lead to different survival probability at the solar surface, after experiencing the resonancel.

Here I show some updated results 24 of our previous fit 23 . I present in Fig. 3 and 4 the allowed parameter region by the rates only and by the combined fit of rates, SK zenith angle dependence and SK spectrum assuming NSNI with $d$-quark and $u$-quark, respectively.
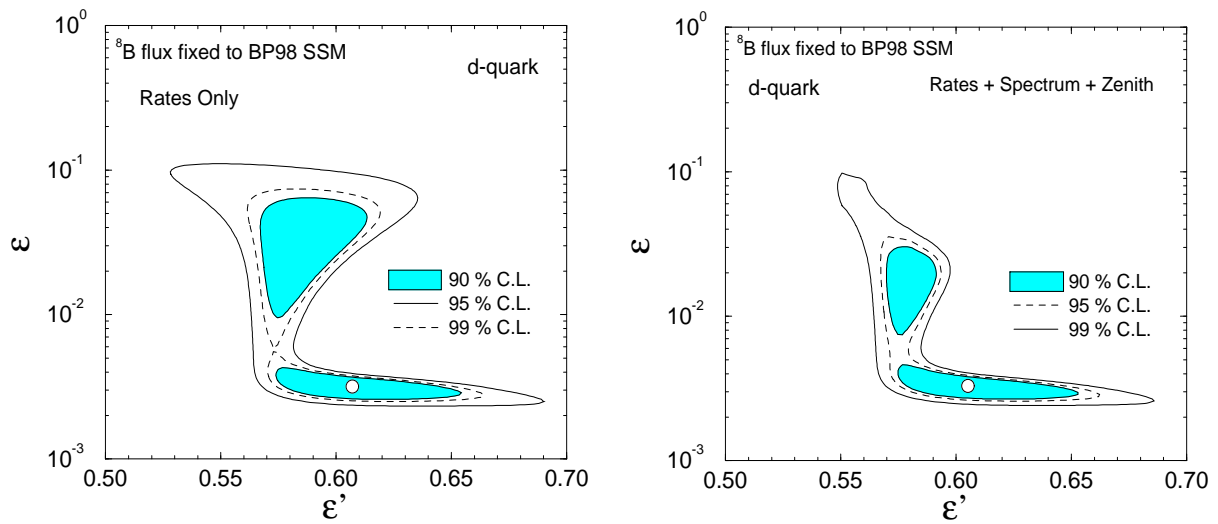

Fig. 3: Allowed parameter region by the rates only (left panel) and the combined data (right panel) assuming NSNI with d-quark. Best fit points are indicated by open circles. Adopted from Ref. 24.
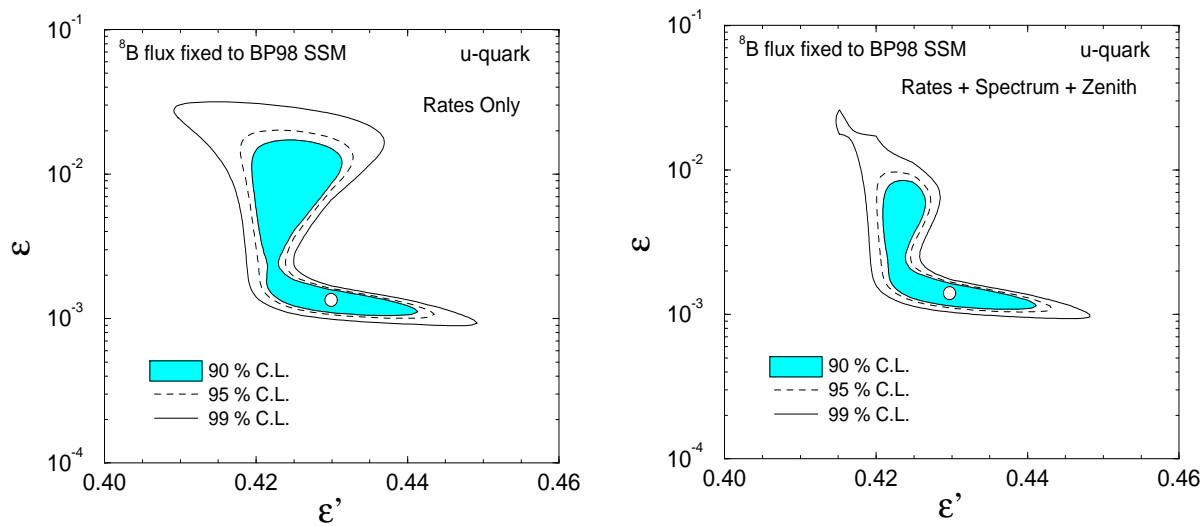

Fig. 4: Same as in Fig. 3 but for u-quark interactions. Adopted from Ref.24. 
It is found that $\chi^{2}=1.67$ for $2 \mathrm{DOF}$ for the rates only and $\chi^{2}=19.6$ for 24 DOF for the combined fit for the case of $d$-quark and $\chi^{2}=1.68$ for 2 DOF for the rates only and $\chi^{2}=19.7$ for 24 DOF for the combined fit for the case of $u$-quark. I conclude that the solar neutrino data can be well accounted for by this mechanism. In Ref. 23 it is discussed that values of $\epsilon$ and $\epsilon^{\prime}$ required to have a good fit are still allowed by the laboratory experiments.

\section{Violation of Equivalence Principle}

Finally, let me consider a even more exotic scenario. The interesting idea that gravitational forces may induce neutrino mixing and flavor oscillations, if there exist violation of equivalence principle (VEP), was proposed about a decade ago 13 , and thereafter, many authors have investigated the possibility of solving the SNP by such VEP induced neutrino oscillations 25 26. It is known that the solution to the SNP can be provided either by the VEP induced MSW-like resonant conversion 25 or by the VEP induced vacuum oscillation 26 . Here, I consider only the latter solution since the parapeter required for the former one can be excluded by a laboratory experiment 27. I will show some updated results 28 of our previous analysis 20 .

Following the framework proposed in Refs.13, to describe the VEP induced massless neutrino oscillation, phenomenologically, we can simply do the following replacement in the usual mass induced oscillation formula: $\Delta m^{2} / 2 E \rightarrow$ $2 E|\phi \Delta \gamma|$ and $\theta \rightarrow \theta_{G}$, where $\Delta m^{2}$ is the mass squared difference, $\phi$ is the gravitational potential which is assumed to be constant in our work as it may come from the local Super-cluster 29, $\theta$ is the usual mixing angle which relate weak and mass eigenstates and $\theta_{G}$ is the mixing which relates weak and gravitational eigenstates, and $\Delta \gamma$ is the quantity which measures the magnitude of VEP. See Ref. 30 for some discussions on possible origins of VEP.

The distinctive feature of this oscillation mechanism, compared to the usual mass induced one, is that the oscillation wavelength $\lambda$ is inversely proportional to the neutrino energy, $\lambda \propto E^{-1}$. This energy dependence is very crucial in obtaining a good fit to the total rates without causing any problem to the fit of the SK spectrum 26, contrary to the situation in the case of usual mass induced vacuum oscillation solution to the SNP 31 .

Here I show some results 28 updated from our previous analysis 26 . I present in Fig. 5 the allowed parameter region in the $\sin ^{2} 2 \theta_{G}-|\phi \Delta \gamma|$ plane, determined only by the total rates (left panel) the allowed region determined by the SK spectrum only (right panel). In Fig. 6, in the left panel I present the allowed region for the combined fit of the rates and the spectrum and also I show in the right panel, the predicted spectra for the best fitted parameters, which are in good agreement with the data. 
It is found that $\chi_{\min }^{2}=1.78$ for 2 DOF for the total rates and $\chi_{\min }^{2}=$ 17.7 for $24 \mathrm{DOF}$ for the combined analysis. I conclude that VEP induced longwavelength oscillation can also provide a good fit to the solar neutrino data, provided that $\Delta \gamma \sim 10^{-20}$ assuming $\phi \sim 3 \times 10^{-5}$ for the local super-cluster 29.
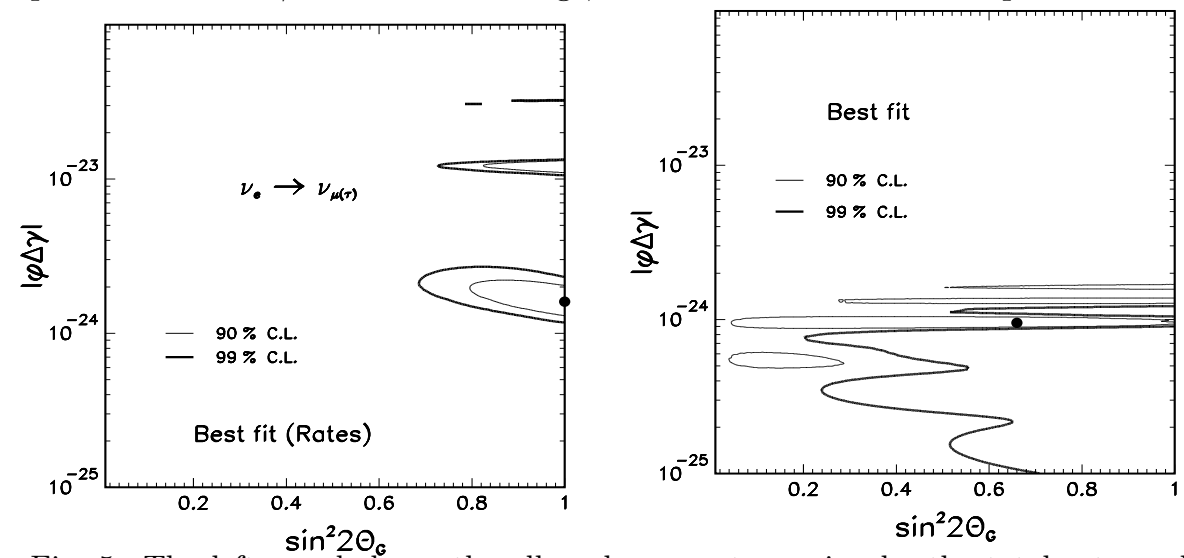

Fig. 5: The left panel shows the allowed parameter region by the total rates only and the right panel shows the allowed region by the SK recoil electron spectrum only where it is the inner part of the contours which is excluded by the SK-spectrum. The best fit points are indicated by the filled circles. Adopted from Ref. 28 .
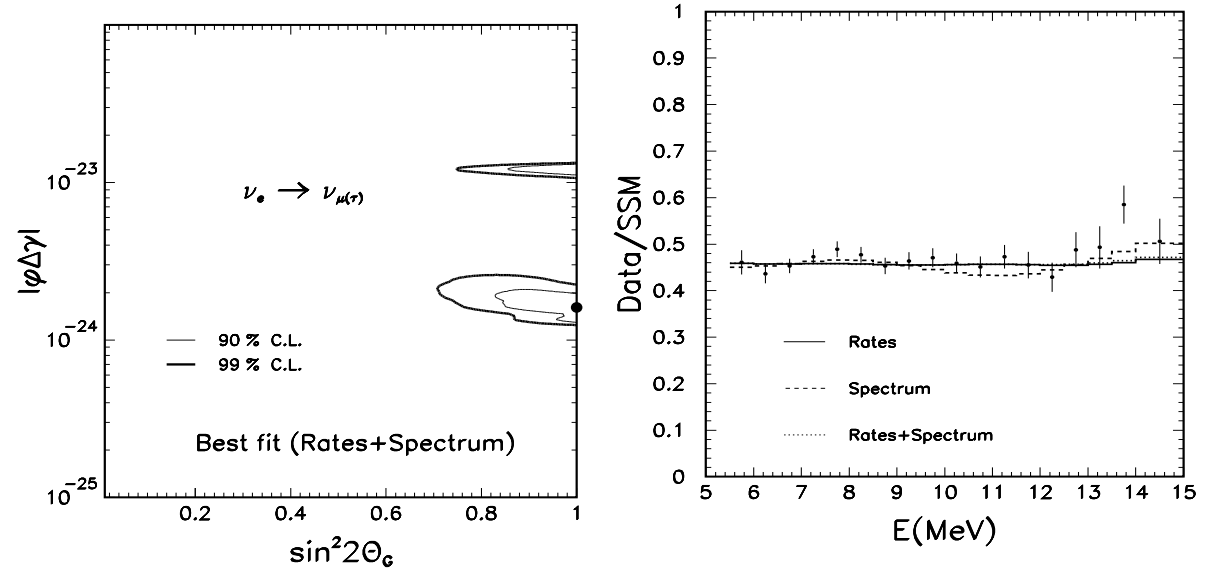

Fig. 6: The left panel is the same as in Fig. 5 but for the rates and SK spectrum combined and the right panel shows the expected SK spectrum for the best fit points. Adopted from Ref.28. 


\section{Implications for Low Energy Solar Neutrinos}

Let me now consider some simple implications for low energy solar neutrines experiments, such as Borexino 32, KamLAND 33, Heron 34, Xmass (Xenon) 35 and GENIUS 36 which aim to observe ${ }^{7} \mathrm{Be}$ and/or $p p$ neutrinos by means of $\nu_{e} e^{-}$scattering reactions.

Let me first define the following quantity,

$$
R(X) \equiv \frac{\text { expected \# of } X \nu \text { events w oscillation }}{\text { expected\# of } X \nu \text { events w/o oscillation }},
$$

where $X={ }^{7}$ Be or $p p$. Explicitly, $R(X)$ can be given by,

$$
R(X) \equiv \frac{\int d E_{e} \int d E_{\nu} \phi_{X}\left(E_{\nu}\right)\left(\frac{d \sigma_{\nu_{e}}}{d E_{e}} P_{e e}\left(E_{\nu}\right)+\frac{d \sigma_{\nu_{\alpha}}}{d E_{e}}\left[1-P_{e e}\left(E_{\nu}\right)\right]\right)}{\int d E_{e} \int d E_{\nu} \phi_{X}\left(E_{\nu}\right) \frac{d \sigma_{\nu_{e}}}{d E_{e}}},
$$

where $E_{e}$ is the recoil electron energy, $\phi_{X}\left(E_{\nu}\right)$ is the neutrino energy distribution of $X$ solar neutrinos, $d \sigma_{\nu_{e, \alpha}} / d E_{e}$ is $\nu_{e, \alpha} e^{-}(\alpha=\mu, \tau)$ scattering cross sections and $P_{e e}\left(E_{\nu}\right)$ is the $\nu_{e}$ survival probability. For simplicity, I do not take into account the resolution function in this contribution.

Next let me try to derive some relation that $R(p p)$ and $R\left({ }^{7} \mathrm{Be}\right)$ must satisfy. The only ongoing experiment which can detect these neutrinos (but not separately) is the ${ }^{71} \mathrm{Ga}$ experiment. The contribution of neutrinos to ${ }^{71} \mathrm{Ga}$ experiment from different reaction sources is expressed as 37 ,

$$
S_{G a} \simeq 69.6\left\langle P_{e e}(p p)\right\rangle+34.4\left\langle P_{e e}\left({ }^{7} \mathrm{Be}\right)\right\rangle+12.4\left\langle P_{e e}\left({ }^{8} \mathrm{~B}\right)\right\rangle+\ldots . \mathrm{SNU},
$$

where $\left\langle P_{e e}(X)\right\rangle\left(X=p p,{ }^{7} \mathrm{Be},{ }^{8} \mathrm{~B}\right)$ indicate the survival probabilities of $X$ neutrinos and I ignored some other minor contributions. Using the relations, $R(X) \sim\left\langle P_{e e}(X)\right\rangle+r(X)\left[1-\left\langle P_{e e}(X)\right\rangle\right]$, where $r(X)$ denotes the ratio of the cross sections $\left\langle\sigma_{\nu_{\mu, \tau} e}\right\rangle /\left\langle\sigma_{\nu_{e} e}\right\rangle$ appropriately averaged over the energy spectrum of $X$ neutrinos, and the observed results of ${ }^{71} \mathrm{Ga}$ experiment, $S_{G a}^{o b s} \simeq 75(1 \pm 0.1)$ SNU, I obtain,

$$
R\left({ }^{7} \mathrm{Be}\right)+2 R(p p) \sim 2 \pm 0.2 .
$$

This is the condition which must be satisfied by any acceptable solution in order to account well for the measurement of the ${ }^{71} \mathrm{Ga}$ experiment.

Now let me try to predict the ranges of $R(p p)$ and $R\left({ }^{7} \mathrm{Be}\right)$ for the various solutions to the solar neutrino problem I discussed in the previous sections. I try to "map" the $95 \%$ C.L. allowed parameter region of each solutions into the plane spanned by $R(p p)$ and $R\left({ }^{7} \mathrm{Be}\right)$. I present in Fig. 7 the expected range of $R(p p)$ and $R\left({ }^{7} \mathrm{Be}\right)$ for various solutions. For the purpose of comparison, I also plot the expected range for the standard mass induced oscillation solutions, 
namely, MSW large mixing angle (LMA), MSW small mixing angle_SMA), MSW low- $\Delta m^{2}$ (LOW) 323.3 and vacuum oscillation (VAC) solutions 31 to the SNP. We can confirm that, roughly speaking, all the solutions satisfy the above relation in Eq. (5).

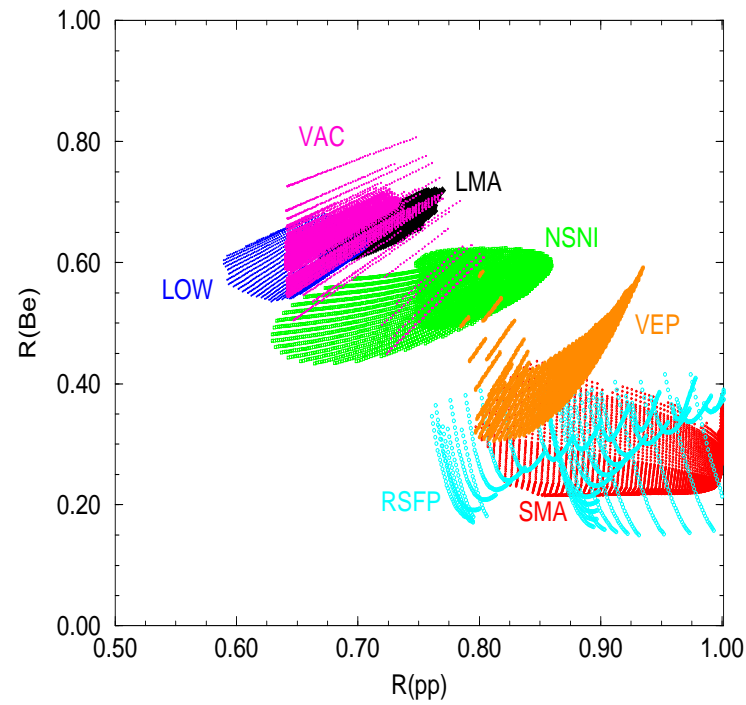

Fig. 7: Predictions for the total rates for $p p$ and ${ }^{7} \mathrm{Be}$ neutrinos for various solutions, which are determined from the $95 \%$ C.L. allowed parameter regions.
As far as rates of $p p$ and ${ }^{7} \mathrm{Be}$ neutrinos are concerned, LMA, LOW and VAC solutions have significant overlap. Similarly, RSFP and SMA have also large overlap. On the other hand, VEP and NSNI solutions have less overlap with the others. From these observations, I can say that LMA, LOW and VAC solutions could be easily confused and the same applies to RSFP and SMA solutions if we will use only the information of $R(p p)$ and $R\left({ }^{7} \mathrm{Be}\right)$. By combining some other information such as zenith angle dependence, time variations, or spectrum distortion, etc., we can discriminate some of

these solutions from the standard mass induced oscillation solutions $1823,263$.

\section{Conclusions}

I showed that alternative solutions to the solar neutrino problem, which do not invoke neutrino mass and/or flavor mixing, still exist and they can provide as good fit as the standard oscillation explanations such as the MSW and VAC solutions to the SNP. While these non-standard solutions are theoretically less motivated than the standard ones which are based only on neutrino mass and mixing, let me stress that it would be important to exclude such nonstandard solutions experimentally, in order to clearly establish the standard solutions such as the MSW ones. I also considered some possible implications for the future low energy solar neutrino experiments and sketched some general features focusing only on the total rates. More detailed and careful comparisons of these solutions as well as some further implications for future solar neutrino experiments will be done elsewhere 39 . 


\section{Acknowledgments}

I would like to thank the organizers of the workshop for invitation. I am grateful to S. Bergmann, A. M. Gago, M. M. Guzzo, P. I. Krastev, P. C. de Holanda, R. Zukanovich Funchal for collaborations and D. V. Ahluwalia, V. Berezinsky, H. Minakata, C. Peña-Garay, O. L. G. Peres, Y. Suzuki, J. W. F. Valle, F. Vissani for valuable discussions. I thank A. M. Gago, P. C. de Holanda and R. Zukanovich Funchal for their kind help in preparing this contribution. The author was supported by the Brazilian funding agency FAPESP.

\section{References}

1. Y. Fukuda et al. (Kamiokande collab.), Phys. Lett. B 335, 237 (1994); Y. Fukuda et al. (Super-Kamiokande collab.), Phys. Rev. Lett. 81, 1562 (1998); D. Casper et al. (IMB collab.), ibid. 66, 2561 (1991); R. Becker-Szendy et al., Phys. Rev. D 46, 3720 (1992); W. W. M Allison et al. (Soudan collab.), Phys. Lett. B 391, 491 (1997); M. Ambrosio et al. (Macro collab.), ibid. B 434, 451 (1998).

2. Z. Maki, M. Nakagawa, and S. Sakata, Prog. Theor. Phys. 28, 870 (1962).

3. B. M. Pontecorvo, Sov. Phys. JETP, 26, 985 (1968); ibid. 6, 429 (1958).

4. S. H. Ahn, et al. (K2K collab.), hep-ex/0103001; see also T. Kobayashi in these proceedings.

5. K. Lande et al. (Homestake collab.), Astrophys. J. 496, 505 (1998); Y. Fukuda et al. (Kamiokande collab.), Phys. Rev. Lett. 77, 1683 (1996); V. Gavrin for the SAGE collab., Nucl. Phys. (Proc. Suppl.) B 91, 36 (2001); W. Hampel et al. (GALLEX collab.), Phys. Lett. B 447, 127 (1999); M. Altmann et al. (GNO collab.), ibid. B 490, 16 (2000); Y. Fukuda et al. (SuperKamiokande collab.), Phys. Rev. Lett. 81, 1158 (1998); ibid. 81, 4279 (1998); ibid. 82, 2430 (1999); ibid. 82, 1810 (1999); Y. Suzuki for the Super-Kamiokande collab., Nucl. Phys. (Proc. Suppl.) B 91, 29 (2001).

6. S. P. Mikheyev and A. Yu. Smirnov, Sov. J. Nucl. Phys. 42, 913 (1985); Nuovo Cim. C 9, 17 (1986); L. Wolfenstein, Phys. Rev. D 17, 2369 (1978).

7. C. Athanassopoulos et al. (LSND collab.), Phys. Rev. Lett. 77, 3082 (1996); Phys. Rev. C54, 2685 (1996).

8. C. S. Lim and W. J. Marciano, Phys. Rev. D37, 1368 (1988); E. Kh. Akhmedov, Sov. J. Nucl. Phys. 48, 382 (1988); Phys. Lett. B 213, 64 (1988).

9. M. M. Guzzo, A. Masiero and S. T. Petcov, Phys. Lett. B 260, 154 (1991).

10. V. Barger, R.J.N. Phillips and K. Whisnant, Phys. Rev. D 44, 1629 (1991); P.I. Krastev and J.N. Bahcall, hep-ph/9703267.

11. E. Roulet, Phys. Rev. D 44, 935 (1991).

12. L. Wolfenstein, Phys. Rev. D 17, 2369 (1978), ibid. D 20, 2634 (1979).

13. M. Gasperini, Phys. Rev. D 38, 2635 (1988); ibid. D 39, 3606 (1980); A. Halprin and C. N. Leung, Phys. Rev. Lett. 67, 1833 (1991).

14. M. Lusignoli, Nucl. Phys. (Proc. Suppl.) 100, 250 (2001); O. Yasuda, in these proceedings, hep-ph/0102167. 
15. J. Schechter and J. W. F. Valle, Phys. Rev. D 24, 1883 (1981); ibid. D 25, 283 (1982).

16. C.S. Lim and H. Nunokawa, Astropart. Phys. 4, 63 (1995).

17. E.K. Akhmedov and J. Pulido, Astropart. Phys. 13, 227 (2000); Phys. Lett. B 485, 178, (2000); J. Pulido, hep-ph/0101116; J. Derkaoui and Y. Tayalati, Astropart. Phys. 14, 351 (2001). O.G. Miranda et al., Nucl. Phys. B 595, 360 (2001).

18. M. M. Guzzo and H. Nunokawa, Astropart. Phys. 12, 87 (1999).

19. Particle Data Group: D.E. Groom et al., Eur. Phys. J. C 15, 1 (2000).

20. J. W. F. Valle, Phys. Lett. B 199, 432 (1987).

21. S. Bergmann, Nucl. Phys. B 515, 363 (1998); S. Bergmann and A. Kagan, ibid. B 538, 368 (1999).

22. M. M. Guzzo et al., hep-ph/0012089.

23. S. Bergmann et al., Phys. Rev. D 62, 073001 (2000).

24. M. M. Guzzo, P. C. de Holanda and H. Nunokawa, Nucl. Phys. (Proc. Suppl.) B 100, 62 (2001).

25. J. Pantaleone, A. Halprin, and C. N. Leung, Phys. Rev. D 47, R4199 (1993); J. N. Bahcall, P. I. Krastev, and C. N. Leung, ibid. D 52, 1770 (1995); S. W. Mansour and T. K. Kuo, ibid. D 60, 097301 (1999); H. Casini, J.C. D'Olivo and R. Montemayor, ibid. D 61, 105004 (2000); D. Majumdar, A. Raychaudhuri and A. Sil, ibid. D 63, 073014 (2001).

26. A. M. Gago, H. Nunokawa and, R. Zukanovich Funchal, Phys. Rev. Lett. 84, 4035 (2000).

27. J. Pantaleone, T. K. Kuo, and S. W. Mansour, Phys. Rev. D 61, 033011 (2000).

28. A. M. Gago, H. Nunokawa and, R. Zukanovich Funchal, Nucl. Phys. (Proc. Suppl.) B 100, 68 (2001).

29. I. R. Kenyon, Phys. Lett. B 237, 274 (1990).

30. A. Halprin and C. N. Leung, Phys. Lett. B 488, 398 (1998); M. Fabbrichesi, M. Piai and G. Tasinato, hep-ph/0012227; G. Z. Adunas, E. Rodriguez-Milla, D. V. Ahluwalia, Gen. Rel. Grav. 33, 183 (2001); Phys. Lett. B 485, 215 (2000).

31. For the analysis of VAC solutions, see for e.g., A. M. Gago, H. Nunokawa and, R. Zukanovich Funchal, Phys. Rev. D 63, 013005 (2001).

32. M. G. Giammarchi, in these proceedings.

33. K. Inoue, in these proceedings.

34. R. Lanou, in these proceedings.

35. Y. Suzuki, in these proceedings, see also hep-ph/0008296.

36. H. V. Klapdor-Kleingrothaus, in these proceedings, hep-ph/0104028.

37. J. N. Bahcall, S. Basu, and M. H. Pinsonneault, Phys. Lett. B 433, 1 (1998).

38. For the analysis of MSW solutions, see for e.g., M. C. Gonzalez-Garcia, P. C. de Holanda, C. Peña-Garay and J. W. F. Valle, Nucl. Phys. B 573, 3 (2000).

39. A. M. Gago et al. in preparation. 